\title{
Comparative Analysis of Feature Extraction Methods for Optic Disc Detection
}

\author{
Harsha Panchariya ${ }^{1}$, Sangita Bharkad ${ }^{2}$ \\ ${ }^{1,2}$ Department of Electronics Engineering, Government Engineering College, Aurangabad, India
}

\begin{abstract}
Medical image analysis and processing has great significance in the field of medicine, especially in non-invasive treatment and clinical study. Normally fundus images are manually graded by specially trained clinicians in a time-consuming and resource-intensive process. A computer assisted fundus image analysis could provide an immediate detection and characterization of retinal features prior to ophthalmologist's inspection. Optic Disc $(O D)$ or Optic Nerve Head $(O N H)$ is relevant anatomical eye structure to diagnose various diseases such as Glaucoma, Cardiovascular diseases, Diabetic retinopathy in its advanced stages. Extraction of $O D$ in retinal fundus image is also helpful in image registration and serves as landmark for other retinal feature localization like macula. This paper gives the brief survey of current OD detection methods with limitations and challenges.
\end{abstract}

Keywords: Diabetic Retinopathy, fundus images, Glaucoma, non-invasive, Optic disc.

\section{Introduction}

Optic Disc connects eye and brain acts as a conduit, transmitting visual information from light sensitive cells of retina to brain. It is also the entry point of major blood vessel that supplies blood to retina [1]. Glaucoma and Diabetic retinopathy are sight threatening diseases which in severe stage causes blindness. So, optic disc detection is an important step for diagnosis of these two diseases as well as some other diseases related to retina. Changes in optic disc shape and area may indicate disease progression. In case of glaucoma detection, the optic cup-to-disc ratio (CDR) determines whether the person is suffering from glaucoma or not. Moreover, in case of Diabetic retinopathy in its advanced stage can be determined by the neovascularization i.e., detection of the new fragile blood vessel in the ONH area. In fundus images optic disc appears as a bright yellowish or white region. It is an entrance of blood vessels and optic nerve to the retina and often serves as a reference for other anatomical features like macula, temporal arcade of main blood vessel. Its shape is approximately circular, interrupted by outgoing vessels. OD is the convergence of blood vessel network and one of the most prominent objects in human retina. It is a vertical oval, with average dimensions of $1.76 \mathrm{~mm}$ horizontally by $1.92 \mathrm{~mm}$ vertically [2]. The usual size of OD is $80-100$ pixels but may vary from person to person.The normal as well as abnormal fundus image consisting of OD is shown in Figure 1. ONH is vertically oval with a peripheral 'rim' composed of neural tissue and blood vessels. It has central depression called as 'Optic cup'. The outermost limit of OD is known as disc margin which can be identified by ophthalmologist as whitish or bright yellowish at the innermost periphery of neuro-retinal rim.

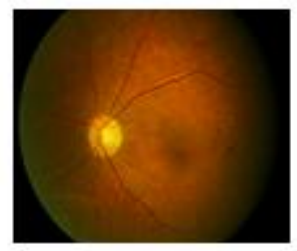

(a)

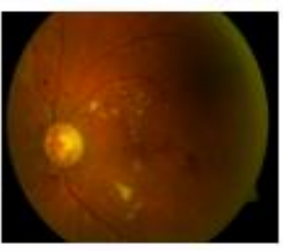

Fig.1. Retinal images (a) normal and (b) abnormal fundus image, with bright ovoid shape as an OD

The detection of the OD in the human retina is a very important task. Because, the optic disc has similar attributes in terms of brightness, color and contrast same as in case of exudate detection in diabetic retinopathy. The optic disc can be seen as a landmark and it can be used for a coarse registration of retinal images in order to reduce the search space for better image. Furthermore, its detection is a first step in understanding ocular fundus images; whether the image is of left eye or right eye. The diameter delivers a calibration of the measurements that determines approximately the localization of the macula, the center of vision, which is of great importance as lesions in the macular region affect vision immediately. Moreover, OD segmentation is key point in finding the various diseases of the retina [3]:

1. Glaucoma detection and diabetic retinopathy detection by changes in CDR and neovascularization. 
2. Patients with headache, sometimes have swollen optic indicates raised intracranial pressure (ICP) which could be due to brain tumor, so presence of brain tumor can be roughly estimated by considering the optic disc shape.

3. Other diseases like cardiovascular diseases, optic neuropathies, optic neuritis and papilledema can be diagnosed with the help of changes in OD appearance.

\section{Feature Extraction Methods For OD Detection}

Even though the OD has well-defined features and a characteristic, localizing the OD automatically and in a robust manner is not as simple since, the appearance of the OD may vary significantly due to retinal pathologies. In order to effectively detect the OD, the various methods developed should consider the variation in appearance, size, and location among different images [4]. The appearance of the yellowish OD region was characterized by a relatively rapid variation in intensity because of the "dark" blood vessels beside the "bright" nerve fibers. So, during segmentation in many cases OD cannot be segmented as whole circular region. In most of the paper researchers considered the OD as the brightest region within retinal image. However, this criterion may not be applicable for retinal images those include other bright regions because of diseases such as exudates due to diabetic retinopathy. Some considered the OD as the area with highest variation in intensity of adjacent pixels. Both the criteria considered by many researchers used their algorithm for normal, healthy retinal images.

OD detection can be classified into various template-based, property-based, transform-based and vessel model-based methods as shown in Fig. 2

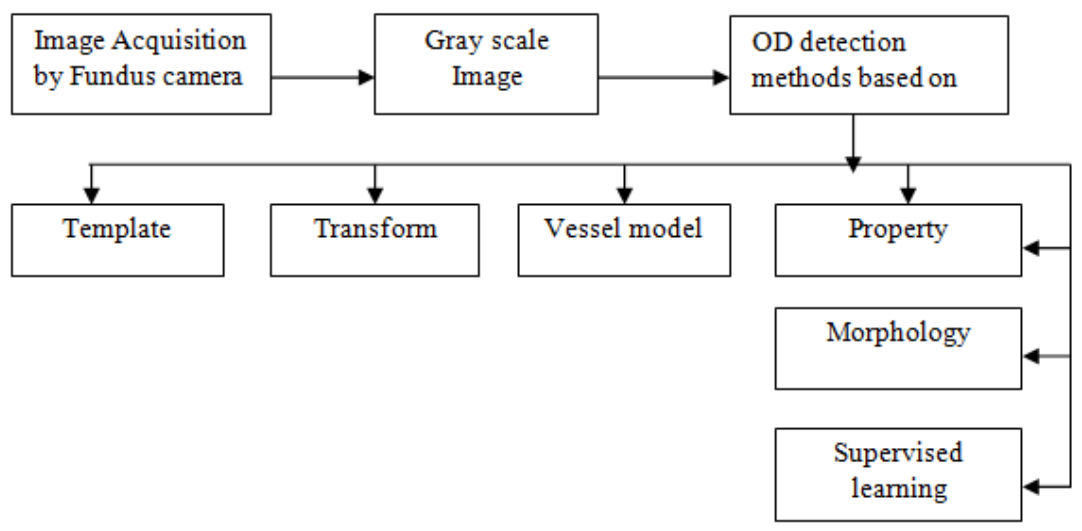

Fig.2. OD detection methods

\subsection{Template-based Method}

Youssif et al. [5] applied template matching to locate the OD center. They first used illumination equalization based and adaptive histogram equalization to smooth the image with a $40 \times 40$ running window. Adaptive histogram equalization and normalization is done to enhance the contrast of an image. Blood vessels are extracted by Matched filter on one scale and 12 directions. Matched Filters are applied to roughly match the direction of vessel in OD. The pixel with least accumulation is considered as OD.

Abramoff and Niemeijer mentioned in the paper [6] that the approach has the potential to detect the location of the OD in retinal images with few or no abnormalities and the position in the image which is located in the middle of the optic disc. To accomplish this they used k-NN regression to find the relationship between the dependent variable d, representing the distance to the optic disc center, and a feature vector measured around a circular template. The template is circular because the optic disc is approximately circular itself. The circular template is placed at various locations in the image and measures the features around the template. Based on the feature vector the distance circular template is used and independent variables $\mathrm{v}$ is measured at the border and under the template. The training set is created storing distance $d$ to true object center. The estimated OD distance $\mathrm{d}, \tilde{d}$ can be calculated by:

$$
\tilde{d}=\frac{1}{k} \sum_{i=1}^{k} d_{i}
$$

Where $d_{i}$ is the stored value of $\mathrm{d}$ of the $\mathrm{i}^{\text {th }}$ nearest neighbor of $\mathrm{v}$ and $\tilde{d}$ location of object of interest that is get detected. The optic disc was considered found when the system indicated a position in the image inside the manually segmented optic disc the average distance between the automatically indicated optic disc center and 
the reference standard was approximately 29 pixels. Lalonde et al. [7] localized OD in low resolution fundus image using Hausdorff based template matching and pyramidal decomposition for tracking OD.

\subsection{Transform-based Method}

Principal component analysis (PCA) has been used by Chutatape and Li [8] to differentiate the optic disc from other main features in retinal images. Brightness guided, PCA model based method was proposed to detect the optic disc as follows:

$$
A A^{T} A v_{i}=\mu_{i} A v_{i}
$$

where $A v_{i}$ is the Eigen vector the subspace defined by $\mu_{i}$ the optic disc is a linear combination of the original training-image vectors and arranged in descending order according to its associated eigenvalue. A fundus coordinate system was established to provide a better description of the features in the retinal images. However, the time consumed for PCA algorithm is more. They used PCA along with active shape model [9] to detect the exact boundary of optic disc which gives better results than their previous work.

Haar [4] applied illumination equalization to the green-band of the RGB fundus image, and then a resolution pyramid using a simple Haar-based discrete wavelet transform was created. Finally, the brightest pixel at the fifth level of the resolution pyramid was chosen to correspond to the OD area. Haar proposed an alternative to the above method based on the pyramidal decomposition of both the vasculature and the greenband, where the fifth level of the resolution pyramid for both the illumination equalized green-band and the binary vessel segmentation are summed, and the highest value corresponds to the OD center. In [10], Ghafar et al. applied the circular Hough transform (CHT) to detect the OD which has a roughly circular shape. The retinal vasculature in the green-band image was suppressed using the closing morphological operator. The Sobel operator and a simple threshold were then used to extract the edges in the image. CHT was finally applied to the edge points, and the largest circle was found corresponds to the OD.

\section{3 .Supervised Learning Method}

Hoover and Goldbaum [11] proposed a method for optic nerve detection based upon an algorithm called fuzzy convergence. This algorithm identified the optic nerve as the focal point of the blood vessel network. Fuzzy convergence uses the endpoints of the blood vessel segments to help find the solution. For every partition $\mathrm{p}$ it uses the following equation for discriminant statistics $F_{p}$ as follows:

$$
F_{p}=\frac{\left(\mu_{A}-\mu_{B}\right)^{2}}{\left(\sigma_{A}^{2}+\sigma_{B}^{2}\right)}
$$

Where $\mu$ and $\sigma$ are the mean and standard deviation of each set. The largest value of indicates the best partition. The method used the convergence of the blood vessel network; this method also takes lot of time to execute on single image.

\section{4 .Vessel model based Method}

Akita et al. [12] localized OD by back-tracking the vessels to their origin. It is one of the good methods to localize OD but the system has to rely on the vascular tree segmentation which is time consuming. Grison et al. [13] proposed a method that is based on a model of the geometrical directional pattern of the retinal vascular system. This system implicitly holds the information on the OD position as the point of vessel convergence. Using the vessel center-line points and the corresponding vessel directions provided by any vessel identification procedure, the model parameters were identified by a means of simulated annealing. These estimated values provide the coordinates of the OD center. Rangayyan et al. [15] detected OD by phase-portrait analysis. Assuming OD is near the focal point of convergence of retinal vessel. The vasculature network is then extracted by Gabor filter. The $\mathrm{ONH}$ is obtained via phase portrait analysis and intensity based condition.

\subsection{Morphology-based Method}

Sopharak et al. [16] used Fuzzy C-means clustering algorithm and then morphological based method to detect OD. First they converted the RGB fundus image into HSI color space image. After preprocessing on I band image of HSI color space image, the morphological closing operation is done using the equation (4):

$$
O P_{1}=\phi^{(B 1)}\left(f_{I}\right)
$$

Where $O P_{1}$ is the closing operation result on I band image $f_{I}$ and $\mathrm{B} 1$ is the structuring element of size 
$B_{1} \in\{6,7,8,9,10\}$ varied accordingly for each image. Then the threshold is applied on closed image with threshold value of $\alpha_{1} \in\left\{0.5,0.6,0.7,0.8,{ }^{\prime}\right.$.The morphological reconstruction by dilation as is performed on previous threshold image as follows:

$$
O P_{3}=R_{f l}\left(O P_{2}\right)
$$

The reconstructed image OP 3 is threshold. If the threshold image consists of exudates then the compactness criteria is applied to reduce the false detection between exudates and OD. In [17], morphological filtering techniques and active contours are used to find the boundary of the optic disc. Thomas et al. [18] have been used mathematical morphology for detection of OD. It is divided into two steps. In first step, the papillary region is localized by exploiting the luminosity of papilla and the vasculature network. This is done because; the gray level variation is higher than any other part of retinal structure. Along with this the shade correction technique is used to remove the background variation. In the second step the boundary is detected by watershed transform using external and internal marker.

\subsection{Property-based Method}

Sinthanayothin et al. [19] detected the OD by identifying the area with the highest average variation among adjacent pixels using a window size equal to that of the OD. The images were preprocessed using an adaptive local contrast enhancement method which was applied to the intensity component. But this algorithm works in average when retinal lesions are present.

$$
\left(i_{d}, j_{d}\right)=\arg \max \left(p_{(i, j)}\right)
$$

Where $\left(i_{d}, j_{d}\right)$ the center of detected OD is, the maximum variance of sub-image $\left(p_{(i, j)}\right)$. B. Zhang et al. [20] proposed a method. In which, first of all multi-scale Gaussian filtering is done on fundus image. Scale production, and double threshold is done to initially extract the vessel's directional map of various thicknesses. The map is then thinned before another threshold is applied to remove pixels with low intensities. In the second step, a Vessels Directional Matched Filter (VDMF) of various dimensions is applied to the candidates to be matched, and the pixel with the smallest difference designated the OD center. Flow diagram for multi-scale Gaussian filtering based OD detection method is shown in Figure 3. Multi-scale Gaussian filter is represented by equation (7):

$$
f_{i}(x, y)=\frac{1}{\sqrt{2 \pi s_{i}}} \exp \left(-\frac{x^{2}}{2 s_{i}^{2}}\right)-m,|x| \leq t . s_{i},|y| \leq \frac{L_{i}}{2}
$$

Where $f(x, y)$ is the threshold image, when green plane image $I_{(x, y)}$ is passed through the 2D-Gaussian curve. $S_{\mathrm{i}}$ represents the scale of the filter and $\mathrm{m}$ is given by:

$$
m=\left(\int_{-t s}^{t s}\left(\frac{1}{\sqrt{2 \pi s_{i}}}\right) \exp \left(\frac{-x^{2}}{2 s_{i}^{2}}\right) d x\right) /\left(2 t s_{i}\right)
$$

is used to normalize the mean value of the filter to 0 . So that the smooth background can be removed after filtering. $L_{i}$ is the length of the neighborhood along the $y$-axis to smooth noise and $t$ is a constant.

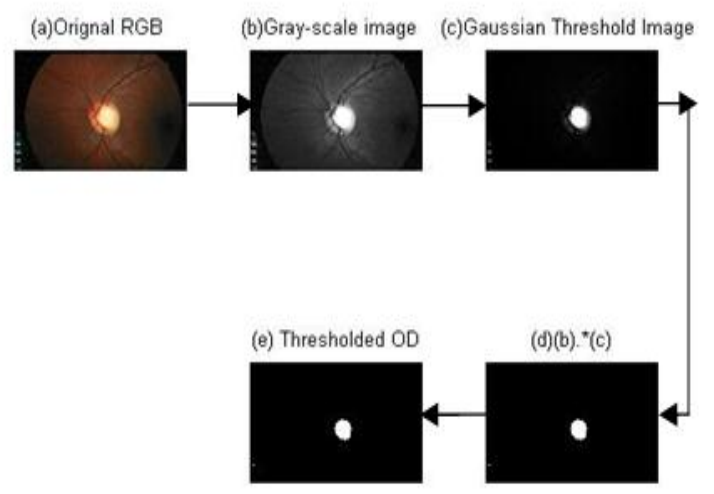

Fig.3. Flow diagram for multi-scale Gaussian filtering based OD detection method 
In [14], Chrástek et al. applied an averaging filter to the green band image of RGB fundus image, and located the OD roughly at the point of the highest average intensity. Koozekanani et al. [21] detected OD by Minimum intensity Maximum Solidity method. They assumed that OD should be typically at the intersection of the reddest and one of the brightest region of fundus image. Summation of the individual intensities with bright pixel as 1 and dark red pixel as 0 is taken. Thus, OD will be detected by intersection with minimum pixel intensity and maximum solidity, where solidity is measure of compactness. Table I shows comparative success rate results of existing methods with different databases.

Table I Comparative Success rate analysis for existing methods of OD detection

\begin{tabular}{|l|l|l|l|l|}
\hline Sr. no & OD detection Methods & Local Database & $\begin{array}{l}\text { STARE } \\
\text { Database }\end{array}$ & $\begin{array}{l}\text { DRIVE } \\
\text { Database }\end{array}$ \\
\hline 1. & Template matching and matched filter [5] & $100 \%$ & $98.8 \%$ & - \\
\hline 2. & Circular template matching and k-NN regression [6] & $99.9 \%$ & - & - \\
\hline 3. & Hausdorff based template matching [7] & $100 \%$ & $71.6 \%$ & - \\
\hline 4. & PCA based method [8] & $99 \%$ & - & - \\
\hline 5. & Haar based DWT [4] & $89 \%$ & $70.4 \%$ & - \\
\hline 6. & Pyramidal decomposition [4] & $93.2 \%$ & $54.3 \%$ & - \\
\hline 7. & Fuzzy Convergence [11] & $97.4 \%$ & $65.4 \%$ & \\
\hline & Geometric parametric model [13] & - & - \\
\hline 8. & Gabor Filter and phase portrait analysis [15] & - & $69 \%$ & $100 \%$ \\
\hline 9. & Morphology based [18] & $100 \%$ & $58 \%$ & - \\
\hline 10. & Variation of Intensity [19] & $99.1 \%$ & $42 \%$ & - \\
\hline 11. & Gaussian filtering [20] & $99.25 \%$ & - & - \\
\hline 12. & Minimum intensity maximum solidity [21] & $99.1 \%$ & $98.68 \%$ \\
\hline
\end{tabular}

\section{* (-) indicates no information is provided in relative field}

\section{Conclusion}

Various methods for locating the OD in retinal fundus image are presented in this paper. An overview of these results shows that some of the existing methods reach better overall performance; some of them provides the shortest computational time and gives better efficiency. But these all methods are not robust as the OD segmentation suffers from many problems like erroneous detection along with exudates, insufficient results in noisy images, in poor contrast and in uneven-illumination. The most challenging part of OD segmentation is preprocessing as each fundus image taken vary by contrast, color etc. The existing algorithm does not work well for the entire standard databases which are publically available. Thus, OD segmentation in medical imaging is the challenging job for the researchers preprocessing the original image before further processing on it is key step in detection of OD. Better preprocessing gives the OD segmentation results up to the mark. Hence there is a need of OD detection algorithms which gives the better performance on noisy databases.

\section{References}

[1] Daniel D. Chiras. Human Biology. Jones and Bartlett Learning, $7^{\text {th }}$ edition, 2011.

[2] H.Ying, M.Zhang and J.-C Liu.,"Fractal-based automatic localization and segmentation of optic disc in retinal images," in Proc. of IEEE Conf. on Engineering in Medicine and Biology Society(EMBS).,2007

[3] http://en.wikipedia.org/wiki/Fundus_photography

[4] F. Haar, "Automatic localization of the optic disc in digital colour images of the human retina," M.S. thesis, Utrecht University, Utrecht, The Netherlands, 2005.

[5] A.Youssif, A Ghalwsh, and A Ghoneim, "Optic disc detection from normalized digitl fundus images by means of vessels' direction matched filter," in IEEE Trans. on Medical Imaging, vol. 27, no. 1, pp. 11-18, 2008.

[6] M. D. Abramoff, Meindert Niemeijer, "The automatic detection of the optic disc location in retinal images using optic disc location regression," in Proc. of IEEE Conf. on Eng Med Biol Soc, pp. 4432- 4435, 2006.

[7] M. Lalonde, M. Beaulieu, and L. Gagnon, "Fast and Robust Optic Disc Detection Using Pyramidal Decomposition and HausdorffBased Template Matching,"in IEEE Trans.On Medical Imaging,vol. 20, no. 11, Nov. 2001.

[8] H. Li and O. Chutatape, "Automatic location of optic disc in retinal images," in Proc. IEEE Conf. on Image Processing.,vol. 2,pp. 837-840. Oct. 7-10, 2001 .

[9] H.Li and O.Chutatape,"Automated Feature Extraction in Color Retinal Images by a Model Based Approach," in IEEE Transaction on Biomed. Engineering, vol.51,No.2, Feb. 2004.

[10] R. A. Abdel-Ghafar, T. Morris, T. Ritchings, and I. Wood, "Detection and characterisation of the optic disk in glaucoma and diabetic retinopathy," in IEEE Conf. on Med. Image Understand Annual, London, U.K., Sep. 23-24,2004.

[11] A. Hoover and M. Goldbaum, "Locating the optic nerve in a retinal image using the fuzzy convergence of the blood vessels," in IEEE Trans. on Med. Imaging., vol.22,no.8,pp.951-958,Aug.2003.

[12] K. Akita and H. Kuga,“ Pattern recognition of blood vessel networks in ocular fundus images," in Proc.of IEEE conf., pp 436441.,1982.

[13] M.Foracchia, E.Grison, A.Ruggeri, "Detection of Optic dics in retinal images by means of geometrical model of vessel structure," in IEEE Trans.on Medical Imaging, vol.23. no10.pp.1189-1195,2004.

[14] R. Chrástek, M. Wolf, K. Donath, G. Michelson, and H. Niemann, “Optic disc segmentation in retinal images,” Bildverarbeitung für die Medizin, Publisher-Springer Berlin Heidelberg . pp.263-266,2002. 
[15] R.Rangayyan, X.Zhu, Anna L. Ells, "Detection of the Optic Nerve Head in Fundus Images of the Retina with Gabor Filters and Phase Portrait Analysis, ”J Digit Imaging, 23(4): pp. 438-453, Aug. 2010.

[16] A.Sopharak, B. Uyyanonvara, S.Barman, T.H William, "Automatic detection of diabetic retinopathy exudates from non-dilated retinal images using mathematical morphology methods," in Computerized Medical Imaging and Graphics(32).pp-720-727 vol. 32,Dec.2008.

[17] F. Mendels, C. Heneghan, and J.-P. Thiran, "Identification of the optic disk boundary in retinal images using active contours," in Proc. of IEEE Conf. on Irish Machine Vision Image Processing Conference. (IMVIP’99), pp. 103-115.Sept-1999.

[18] T. Walter, J.C Klein, P. Massin, and A. Erginay, "A Contribution of Image Processing to the Diagnosis of Diabetic RetinopathyDetection of Exudates in Color Fundus Images of the Human Retina," in IEEE Trans.Med.Imag., Vol.21 no.10, Oct. 2002.

[19] C. Sinthanayothin, J. F. Boyce, H. L. Cook, and T. H.Williamson, "Automated localisation of the optic disk, fovea, and retinal blood vessels from digital colour fundus images,” Br. J. Ophthalmol., vol. 83, no.8,pp. 902-910,1999.

[20] B.Zhang, J.You,F.Karray, "Optic Disc Detection by Multi-scale Gaussian Filtering with Scale Production and a Vessels' Directional Matched Filter," in Proceedings of Second International Conference, ICMB 2010, Hong Kong, China, June 28-30, 2010.

[21] S.Roychowdhury, D. Koozekanani,K. Parhi, "Screeening Fundus Images for Diabetic Retinopathy," in Prco of IEEE conf. Signals, Systems and Computers (ASILOMAR),pp-1641-1645. Nov.2012. 